\title{
REVIEW \\ Competition as a source of constraint on life history evolution in natural populations
}

\begin{abstract}
AJ Wilson
Competition among individuals is central to our understanding of ecology and population dynamics. However, it could also have major implications for the evolution of resource-dependent life history traits (for example, growth, fecundity) that are important determinants of fitness in natural populations. This is because when competition occurs, the phenotype of each individual will be causally influenced by the phenotypes, and so the genotypes, of competitors. Theory tells us that indirect genetic effects arising from competitive interactions will give rise to the phenomenon of 'evolutionary environmental deterioration', and act as a source of evolutionary constraint on resource-dependent traits under natural selection. However, just how important this constraint is remains an unanswered question. This article seeks to stimulate empirical research in this area, first highlighting some patterns emerging from life history studies that are consistent with a competition-based model of evolutionary constraint, before describing several quantitative modelling strategies that could be usefully applied. A recurrent theme is that rigorous quantification of a competition's impact on life history evolution will require an understanding of the causal pathways and behavioural processes by which genetic (co)variance structures arise. Knowledge of the G-matrix among life history traits is not, in and of itself, sufficient to identify the constraints caused by competition.
\end{abstract}

Heredity (2014) 112, 70-78; doi:10.1038/hdy.2013.7; published online 27 February 2013

Keywords: indirect genetic effects; evolutionary constraint; behaviour; personality; G-matrix

\section{INTRODUCTION}

Classical quantitative genetic models tell us that a heritable phenotypic trait under selection should evolve. In the simplest case-where selection acts on a single trait only-the change in phenotypic mean after one generation of selection $(R)$ is predicted by the breeders equation (Lush, 1937) as the product of the narrow sense heritability $\left(h^{2}\right)$ and a linear selection differential $(S)$. Over the past two decades there has been tremendous interest in estimating these (and other) quantitative genetic parameters in wild-animal populations, as a step towards a better understanding of how phenotypes evolve under natural selection (Kingsolver et al., 2001; Ellegren and Sheldon, 2008; Kruuk et al., 2008). A pattern that has emerged from this work is that linear (directional) selection on traits is common (Kingsolver et al., 2001; Kingsolver and Diamond, 2011), while heritable variation for fitness-related traits is also widespread (Kruuk et al., 2008). Although it is important to acknowledge that the difficulty of accurately and precisely estimating selection and genetic variance in wild populations can be great (Stinchcombe et al., 2002; Kruuk and Hadfield, 2007; Hadfield, 2008; Morrissey et al., 2010), on taking parameter estimates at face value phenotypic stasis is commonly observed in situations where relatively rapid trait evolution is predicted (Merilä et al., 2001). This has given rise to the interest in the question of what constrains phenotypic evolution (Merilä et al., 2001; Kruuk et al., 2008; Blows and Walsh, 2009).

That the univariate breeder's equation is not adequate for predicting phenotypic change in a natural population should not surprise us. It is only a complete description of phenotypic change under particular conditions (for example, a constant environment, negligible genetic drift and discrete generations) and makes assumptions that are probably untenable in any natural population (for example, that selection is not acting on traits genetically correlated with the character of interest). Nonetheless, it provides a useful yardstick against which to test biological hypotheses about the origin of evolutionary constraint in any given case (Morrissey et al., 2010). Here the term constraint will be used throughout to describe any process that reduces the rate of evolutionary change relative to naive expectations from the simple models. Fluctuating selection, maternal effects, interlocus conflict, $G \times E$ interactions and selection on genetically correlated traits are all potential sources of constraint that are being subjected to empirical scrutiny by evolutionary biologists and ecologists (Fairbairn and Roff, 2006; Wilson et al., 2006; Siepielski et al., 2009). However, one hypothesised source of constraint on phenotypic evolution that has received very limited empirical scrutiny to date is competition.

Competition among individuals, which occurs when one or more resource (for example, food, territory, mating opportunities) is limiting, is central to our understanding of ecology and population dynamics. However, it also has major implications for the evolution of phenotypic traits both as a source of selection (for example, malemale competition selecting for weaponry traits) and because it can influence the genetic (co)variance structure of resource-dependent life history traits (for example, growth, maturation, fecundity). When competitive interactions occur, the expression of a resourcedependent phenotype, for example, growth, in any focal individual will depend on the extent to which its resource acquisition is decreased by its competitors. Under conditions of resource 
limitation, we expect a reduction in mean growth rate (due to a reduction in mean resource acquisition). However, if individuals differ in competitive ability, then winners will gain (relatively) more resource at the expense of losers and we might also expect an increase in phenotypic variance. Viewing competition as a purely environmental effect, then all else being equal (for example, additive genetic variance remains constant, the strength of selection is unchanged), an increase in population size and/or decrease in total resource might reduce heritability and thus the rate of trait evolution (Charmantier and Garant, 2005).

However, the view that competition contributes only environmental variance may be overly simplistic if among-individual variation in competitive ability itself has a genetic component (Bijma et al., 2007; Bijma and Wade, 2008; Hadfield et al., 2011). In this case, genetic influences on focal phenotype can arise not just from an individual's own genes (direct genetic effects), but also from genes present in the competitive environment provided by others-so-called associative (Griffing, 1967, 1976) or indirect genetic effects (IGEs; Moore et al., 1997). Broadly defined as occurring any time that the phenotype of one individual is causally influenced by the genotype of another, IGEs actually have wide-ranging implications that cut across disparate fields of pure and applied research. For example, a growing recognition that observed phenotypes can be under shared genetic control is providing new insights into the evolution of social traits, including aggression (Moore et al., 2002; Wilson et al., 2009a), parental care (Kolliker et al., 2005) and cooperation (McGlothlin et al., 2010). In a very different context, incorporation of IGEs into artificial selection schemes offers the potential to improve target traits (Bergsma et al., 2008; Cappa, 2008) while simultaneously improving welfare in livestock species by reducing the expression of behavioural aggression (Muir and Craig, 1998; Ellen et al., 2007). Here I pose the question: are IGEs an important source of evolutionary constraint for resourcedependent traits in natural populations? Existing theory tells us that they could be (Hadfield et al., 2011), but we currently have little idea of how prevalent or large genetically determined competitive effects are. Consequently, we cannot know to what extent competitionwhich is fairly ubiquitous in natural populations - may contribute to the frequent observation of phenotypic stasis for heritable traits under directional selection (Cooke et al., 1990; Merilä et al., 2001). A major objective of this article is to highlight the need for empirical research that will help us to answer this question.

In what follows I first provide a brief overview of the existing theory to explain how, and why, competition could impose currently unrecognised constraints on phenotypic evolution. I then discuss a number of emergent patterns from life history studies of wild animals, and argue that -at least in some cases - the empirical data are actually more consistent with an IGE-based model of constraint than with the classical view that life history evolution is constrained by trade-offs (Stearns, 1989). Finally, I outline the key hypotheses that need testing, and attempt to provide some practical suggestions as to where (that is, what sort of systems) and how (that is, useful modelling strategies) this may be achieved. A recurrent theme throughout is that to properly understand the origin of evolutionary constraint, quantification of the (direct) genetic covariance structures among resource-dependent traits may well be insufficient. Rather we will also need to explicitly consider the behavioural traits and pathways that mediate competitive interactions and thus shape these covariance structures. With this in mind I draw attention to several recent developments in behavioural research that could usefully inform quantitative genetic studies of IGEs in general and their contribution to evolutionary constraint in particular.

\section{HOW CAN COMPETITION CAUSE EVOLUTIONARY CONSTRAINT?}

The mechanism by which competition could constrain the evolution of resource-dependent traits relative to predictions from simple models can be explained by a simple example. Imagine a population in which growth rate is resource-limited, heritable and under positive directional selection. A genotype that predisposes to fast growth may do so by increasing the competitive ability, and therefore the resource acquisition, of the bearer. Such genes will have both direct effects (that is, increasing the bearer's growth rate) and indirect effects (that is, reducing resource acquisition by-and therefore growth ofcompeting individuals). Under this scenario, directional selection on individual growth will cause correlated evolution of a more competitive environment. This phenomenon, termed 'evolutionary environmental deterioration' (Fisher, 1958) or the 'treadmill of competition' (Wolf, 2003), arises because as a population evolves a winning lineage finds itself competing against more and more winners in each successive generation (Hadfield et al., 2011). The social environment 'deteriorates' with each generation, a change that offsets the increase in mean growth rate predicted by the breeder's equation (Fisher, 1958; Cooke et al., 1990; Frank and Slatkin, 1992).

This was illustrated formally by Hadfield et al. (2011), who derived an expression for the expected change in the mean of a resourcedependent trait (for example, growth) $\left(y_{1}\right)$ in a population characterised by genetic variance for resource acquisition under competitive conditions, henceforth termed 'competitive ability' $\left(y_{2}\right)$. If individuals acquiring more resource grow faster and have higher fitness, then there will be a positive linear directional selection differential $(s)$ on $y_{1}$. However, if the selection differential is generated entirely by variance in resource acquisition and not by variance in allocation of acquired resource to growth, then using selection gradients ( $\beta$; Lande and Arnold, 1983) to disentangle the direct and indirect targets of selection would yield $\beta_{y 1}=0$, while $\beta_{y 2}>0$. In this case, the expected change in mean growth rate $\Delta_{\overline{y 1}}$ after a single generation of selection is given (following a slight rearrangement of equation 9 of Hadfield et al., 2011) as:

$$
\Delta_{\overline{y 1}}=\Delta \log (A)-\Delta \log (N)+\beta_{y 2}\left(\sigma_{A 2}^{2}+\sigma_{A 1, A 2}\right)-\beta_{y 2} \sigma_{A 2}^{2}
$$

Where $A$ is the total amount of resource being divided among $N$ competing individuals, $\sigma_{A 2}^{2}$ denotes the additive genetic variance in trait 2 and $\sigma_{A 1, A 2}$ denotes the additive covariance between traits 1 and 2 (which is positive). The first two terms simply imply that if total resource $(A)$ increases or population size $(N)$ decreases then average growth rate will go up. (Note that the logarithmic scaling is an incidental consequence of the term's definition in Hadfield et al. (2011), but is retained here for consistency.) The third term in this equation describes the evolutionary change in growth $\left(y_{1}\right)$ expected as a consequence of the direct selection on the genetically correlated trait of competitive ability $\left(y_{2}\right)$ and will be positive (since $\beta_{y_{2}}>0$ ). In fact, under the assumption that only resource acquisition has a non-zero selection gradient, this term is equal to the additive genetic covariance between growth and relative fitness (Hadfield et al., 2011) and can therefore be interpreted as the Robertson-Price identity (Robertson, 1966). In the absence of IGEs, estimation of the Robertson-Price identity provides a valid prediction of the evolutionary change expected for a trait without explicit knowledge of the mechanism of selection (Morrissey et al., 2010). However, with genetic variance in competitive ability this does not hold true. The final term of Equation 1 is negative and describes the reduction in $\Delta_{\overline{y 1}}$ caused by the evolutionary deterioration of the environment. Genetic variance for competitive ability, which implies that IGEs on resource 
acquisition must exist, thus results in less phenotypic evolution than would be predicted from either the breeder's equation or the Robertson-Price identity.

\section{IMPLICATIONS FOR PATTERNS OF COVARIANCE AMONG LIFE HISTORY TRAITS}

If competitive interactions have the potential to be an important source of evolutionary constraint in natural populations, whether they actually are remains an open question. However, it is interesting to note that the causal model of constraint on which Equation (1) is based predicts positive (direct) genetic covariance structure among resource-dependent life history traits. This is in contrast to the negative genetic correlations (with respect to effects on fitness) that are expected if, as is widely assumed in evolutionary ecology, constraint arises primarily from trade-offs among life history traits or fitness components (Stearns, 1989; Roff, 2002). At equilibrium, the covariance structure among resource-dependent life history traits could therefore help to discriminate between these two mechanisms of constraint (with the important caveat that they are not actually mutually exclusive).

Resource allocation trade-offs occur because, for an individual with finite resource, increased allocation to one trait (for example, growth or somatic maintenance required for survival) must come at the expense of another (for example, fecundity). Assuming that two traits $(\mathrm{X}, \mathrm{Y})$ are heritable and defined so as to be under positive selection, then a constraint arises if they are also negatively genetically correlated (Figure 1a). In this scenario, the fitness increase expected by a selection response in the first trait towards its optimum would be offset by a correlated response in the second trait away from its optimum. If selection purges those mutations with deleterious effects on both traits and fixes those with beneficial effects on both traits, segregation of antagonistic genetic variance is predicted at equilibrium. In contrast, if a population is characterised by genetic variance in competitive ability, then an individual with a high genetic merit will be able to acquire more resource than a genetically poor competitor, and thereby allocate more to all traits. Thus, to the extent that genetic variance in resource-dependent traits arises through this competitive pathway, we actually expect positive genetic covariance among them (Figure $1 \mathrm{~b}$ ). In this second scenario, an evolutionary constraint arises not because of the allocation trade-off (though this must still exist at the within-individual level), but because of IGEs and so evolutionary environmental deterioration.

The quantitative genetic basis of trade-offs is assumed more often than tested in evolutionary ecology. Where the genetic correlation $\left(r_{\mathrm{G}}\right)$ has been estimated between traits in putative trade-offs, the predicted negative genetic correlations are often not found (Kruuk et al., 2008; House and Simmons, 2012). In fact, for pairs of life history traits estimated in situ in wild vertebrates, the estimates are overwhelmingly positive (Kruuk et al., 2008). Field-based studies also commonly report positive phenotypic covariance among positively selected life history traits, an observation that is not incompatible with the trade-off model as environmental sources of covariance will often be positive (for example, spatial heterogeneity in resource availability) and large enough to mask underlying genetic relationships (van Noordwijk and de Jong, 1986). However, Cheverud's (1988) conjecture - that the phenotypic correlation $\left(r_{\mathrm{p}}\right)$ provides a reasonable predictor of $r_{\mathrm{G}}$-generally holds true for the sign of a correlation at the meta-analytic level (Roff, 1996; Kruuk et al., 2008; Dochtermann, 2011). Thus, it seems likely these positive covariance patterns are telling us something about the underlying genetic covariance structures. This is not to say that trade-offs are

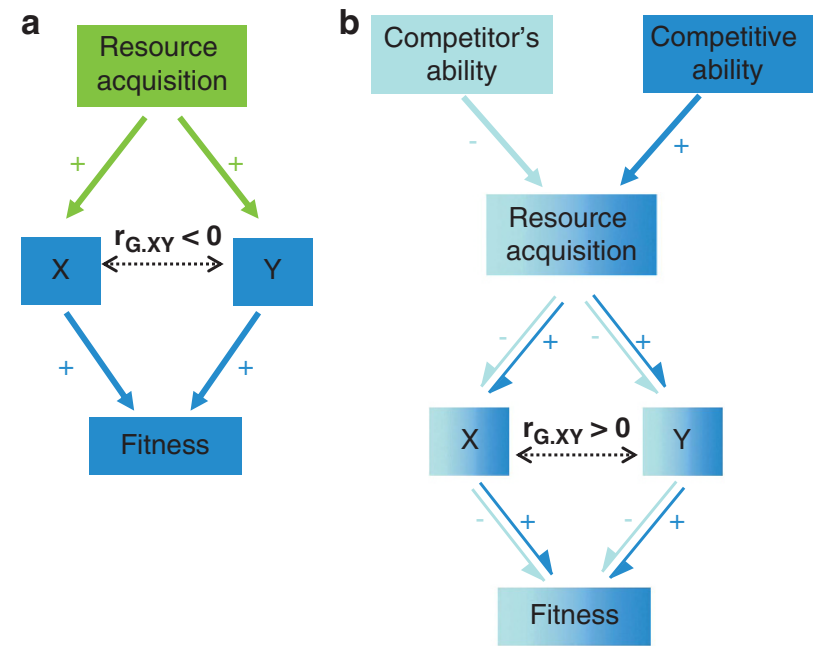

Figure 1 Diagrammatic representation of trade-off (a) and competitionbased (b) models of constraint. Under the trade-off model (a) two heritable (blue) traits $X$ and $Y$ are positively selected but constrained by a negative genetic correlation $\left(r_{\mathrm{G} . X Y}\right)$. Environmental effects (green) on resource acquisition may generate positive covariance between $X$ and $Y$, masking the underlying genetic relationship. In the competition-based model (b), resource acquisition is heritable and also subject to indirect genetic effects (light blue) from competitors. Direct and indirect genetic effects are propagated to downstream traits ( $X$ and $Y$ ) and fitness. IGEs impose a genetic constraint that will not be apparent from considering direct genetic effects alone (as $r_{\mathrm{G} . X Y}>0$ ).

unimportant as a source of evolutionary constraint, only that they may not be as important or ubiquitous as is sometimes believed.

More generally, the limitations of a bivariate approach to understanding evolutionary constraint, as encapsulated by the trade-off concept, have been well highlighted (Charlesworth, 1990; Blows and Walsh, 2009). Given that natural selection is multivariate, studies of genetic constraint should ideally consider the full geometry of the Gmatrix and not just its individual elements (Blows and Walsh, 2009). However, multivariate approaches as currently applied do not allow recognition of competition-based constraint. For example, the scenario depicted in Figure $1 \mathrm{~b}$ could be expanded to include additional resource-dependent traits beyond $\mathrm{X}$ and $\mathrm{Y}$, but estimation of $\mathbf{G}$ and multiplication by the vector of selection $\boldsymbol{\beta}$ according to the multivariate breeder's equation (Lande, 1979) would still yield an upwardly biased prediction of evolutionary change. Nor would inclusion of resource acquisition or competitive ability correct the prediction; the predictive model of change is inadequate here not because of a missing trait, but rather because is does not account for the consequences of IGEs.

In the (unrealistically) simple case that $\mathbf{G}$ among $n$ positively selected resource-dependent traits was determined entirely by (direct) genetic effects on competitive ability, eigen decomposition would reveal eigen values of zero for all but the first vector. Thus, decomposing $\mathbf{G}$ would reveal constraint in the important sense that most directions of multivariate evolution are impossible even if selected for (Blows and Walsh, 2009). However, the first eigen vector of $\mathbf{G}$ would capture the (direct) genetic variance in resource acquisition and be closely aligned with $\boldsymbol{\beta}$. Consequently, this analysis would not explain the lack of phenotypic response to selection as acting. Several recent studies have actually reported evidence for within-population variation in individual 'quality' (Hamel et al., 2009; Moyes et al., 2009), loosely understood as a major axis of 
multivariate phenotypic variance closely aligned with $\boldsymbol{\beta}$ (see Bergeron et al., 2010 and Wilson and Nussey, 2010 for critiques of the lack of formal definition). Quality is therefore the multivariate analogue of finding positive covariance between traits in a putative trade-off, and while it could similarly be explained by environmental variance in resource acquisition (Wilson and Nussey, 2010), in some cases it has been shown to be heritable (Coltman et al., 2005).

\section{TOWARDS AN EMPIRICAL STRATEGY FOR ESTIMATING COMPETITION-BASED CONSTRAINT}

Positive genetic covariance structure among (positively selected) life history traits showing phenotypic stasis is consistent with a competition-based model of evolutionary constraint. However, to directly test the importance of this mechanism investigating the genetic basis of variation in competitive ability is vital. Unfortunately, in situ parameterisation of the model in Equation 1 is difficult. Even in those field systems most amenable to quantitative genetic approaches, phenotyping efforts have typically targeted morphological and life history traits. Consequently, we lack data on individual resource acquisition or suitable proxies for competitive ability (but see, for example, Wilson et al. (2011)). Furthermore, total resource (A) will usually be the unknown meaning that distinguishing evolutionary environmental deterioration from a non-evolutionary decrease in total resource is problematic (Cooke et al., 1990; Hadfield et al., 2011).

Although the genetic architecture of competition has received limited attention to date, several strategies would seem useful. The first is to identify those phenotypic traits that determine an individual's competitive ability and determine whether they are actually heritable. This answers the qualitative question of whether evolutionary environmental deterioration is expected, but cannot quantify the magnitude of the constraint it will impose on life history evolution. The second is to explicitly model IGEs on resourcedependent traits, and predict selection responses using theory that incorporates this source of genetic variance (and, where relevant multi-level selection processes). Given an appropriate data structure IGEs can be modelled with or without explicit knowledge of which trait(s) comprises competitive ability (using so-called 'trait-based' or 'variance component' IGE models, respectively; McGlothlin and Brodie, 2009). A third approach, useful for reasons that are explained in full below, is to compare estimates of the $\mathbf{G}$ matrix, before and after conditioning on social dominance. The best choice of strategy (or combination of strategies) will depend on a number of questionsthe answers to which will vary among empirical systems and contexts. For example, is it possible to observe an individual's competitive ability directly? Can groups of competing individuals be readily defined (in the field) or controlled (in the laboratory)? What is the limiting resource (for example, food, territory, mating opportunities)? What type of behavioural interactions, if any, occur among competitors trying to use that resource?

Contest versus scramble: how can we measure 'competitive ability'? The most direct approach to determining whether environmental deterioration can occur at all is simply to test whether competitive ability is heritable. This requires phenotyping individuals from a wild or experimental population, followed by analysis using a method appropriate to the pedigree structure (for example, parent-offspring regression, sib analysis, individual-based animal model). However, what constitutes an appropriate measure for 'competitive ability' deserves careful consideration and may depend on the ecological form of competition occurring.
Ecologists have long distinguished between 'contest' and 'scramble' forms of competition (de Nicholson, 1954; Jong, 1976), the former occurring when individuals are able to exclude competitors thereby winning exclusive access to a resource (for example, two males fighting to win a harem), the latter occurring when a resource cannot be controlled such that all individuals get some access (for example, a herd of ungulates grazing a food resource). Although these are idealised ends of a continuum, it seems likely that more 'contestlike' scenarios will be more empirically tractable for studies of IGEbased constraint in several respects. First, contest competition commonly occurs through dyadic interactions, the outcome of which is known in many species to depend on clearly defined aggressive behaviours, size or weaponry traits. Behavioural biologists view these traits as contributing to an individual's 'resource holding potential' (Parker, 1974), and they can therefore provide useful proxies of competitive ability. In many cases, these traits are known a priori to be heritable (for example, antler size in red deer; Kruuk et al., 2002). Second, contest outcome (which translates directly into resource acquisition) is easily observed allowing dominance hierarchies to be determined from field observations (Wilson et al., 2011) or through staged contests in a laboratory setting. Third, as winners gain all resource at the expense of losers, there is stronger asymmetry of resource acquisition expected under contest competition than under scramble (Nicholson, 1954). All else being equal, indirect (genetic) effects should therefore contribute more to variance in resourcedependent traits.

In contrast, the traits that determine individual competitive ability under scramble-like competition may be less obvious. Certainly, where food is the limiting resource it is reasonable to expect among-individual variation in feeding rate in the presence of competitors to be informative. This has been shown to be repeatable under field conditions in some species (for example, blackbirds; Cresswell, 2001). More recently, studies in the burgeoning field of animal personality (Réale et al., 2007) are increasingly reporting that 'boldness' - the repeatable tendency of an individual to explore and/or take risks - is positively associated with food intake and life history productivity (Biro and Stamps, 2008). Whether boldness is functionally important for resource acquisition under scramble competition (for example, if bolder individuals more likely to arrive first at a resource) remains to be seen. However, it is worth noting that boldness-related traits can be heritable (Dingemanse et al., 2012) and positively correlated with aggressiveness (Rudin and Briffa, 2012). As aggressiveness is (usually) indicative of success in contest competition, this raises the interesting question of whether populations are characterised by (genetic) variance in competitive ability per se. If so an individual (or genotype) better able to assert dominance in a dyadic contest should also acquire more than average resource under scramble. Within a classical quantitative genetic framework this hypothesis could be examined by testing for (strong) positive genetic correlation structure among those traits known (or assumed) to reflect competitive ability in different contexts.

\section{Trait-based and variance-partitioning IGE models}

There are several recently proposed models of phenotypic evolution that explicitly incorporate IGEs, as well as multilevel selection processes and relatedness structure (Bijma et al., 2007; McGlothlin et al., 2010). These provide a general quantitative genetic framework for modelling social traits and therefore have implications for, and applications to, many topics in evolutionary ecology. IGE models have generally been formulated in one of two ways-termed 'trait-based' and 'variance partitioning' approaches by McGlothlin and Brodie 
(2009). Translation between these two approaches is quite possible, and either can be applied to multivariate phenotypes influenced by interactions among individuals within groups of any size (Bijma et al., 2007; McGlothlin and Brodie, 2009). Here, I highlight how these models could be used by empiricists to study competition, but refer the interested reader elsewhere, for example, Bijma and Wade (2008) and McGlothlin and Brodie (2009), for wider discussion.

Following Moore et al. (1997), a trait-based approach might specify individual is phenotype for a resource-dependent trait (for example, growth, $y_{1}$ ) competing with individual $j$ as:

$$
\mathrm{y}_{1 \mathrm{i}}=\mu_{1}+\mathrm{a}_{1 \mathrm{i}}+\varepsilon_{1 \mathrm{i}}+\psi_{12} \cdot \mathrm{y}_{2 \mathrm{j}}
$$

where $\mu_{1}$ is the population mean growth rate, $a_{1}$ is an individual (direct) additive genetic merit for growth rate, $\varepsilon_{1 \mathrm{i}}$ is an effect of the environment (independent of competition) and $y_{2}$ is an interacting trait' known (or hypothesised) to measure competitive ability. The final parameter $\psi_{12}$ describes the regression of this trait (expressed by $j$ ) on growth (expressed by $i$ ), and will be negative (a more competitive individual $j$ will mean a lower growth rate for $i$ ). If $y_{2}$ is heritable then Equation 2 can be rewritten as

$$
\mathrm{y}_{1 \mathrm{i}}=\mu_{1}+\mathrm{a}_{1 \mathrm{i}}+\varepsilon_{1 \mathrm{i}}+\psi_{12} \cdot \mathrm{a}_{2 \mathrm{j}}+\psi_{12} \cdot \varepsilon_{2 \mathrm{j}}
$$

where $\psi_{12} \cdot a_{2 j}$ is the IGE on $i$ 's growth, which is determined as the product of the $j$ 's direct genetic merit for 'competitive ability' and the regression parameter $\psi$. The simple model of individual phenotype expressed in Equation 3 can be extended in various ways (for example, to account for multiple interacting traits, feedback loops between traits), with selection responses predictable given estimates of the additive genetic (co)variance of traits 1 and 2, $\psi_{12}$, and knowledge of the selection regime (see McGlothlin and Brodie (2009) and Moore et al. (1997) for further details). Importantly, empirical application therefore requires that both the resource-dependent trait $\left(y_{1}\right)$ and competitive ability $\left(y_{2}\right)$ be observed. Valid predictions of evolutionary change are contingent on there being a causal dependence of $y_{1 \mathrm{i}}$ on $y_{2 j}$, which may be difficult to verify in some systems. Furthermore, if an empirical study finds that $\psi_{12}=0$, or that $y_{2}$ is not heritable (implying $a_{2 j}=0$ for all $j$ ), then this does not mean IGEs on growth do not occur, only that they do not operate via trait 2. It is possible that $y_{2}$ is a poor measure of individual competitive ability.

If a suitable proxy for competitive ability is not available, then an alternative strategy lies in modelling the effect (or 'performance') of a particular individual on a trait of interest expressed in focal conspecifics. By comparing the resource-dependent phenotypes of individuals interacting with known competitors, we can statistically determine how much of the phenotypic variance is explained by competitor genotype (that is, IGEs). This is possible if focal and competitor identities are known, and if all individuals are contained within a pedigree structure that spans groups of competing individuals. Following earlier work (Griffing, 1967; Griffing, 1976; Muir, 2005; Bijma et al., 2007) derived expressions for the selection response in $y_{1}$, which depends on total genetic variance (which arises from both direct and indirect effects and is also a function of group size $n$ ), relatedness structure, strength of selection, and the extent to which selection is multilevel (that is, among-groups rather than among-individuals). Presented here with some notational changes for internal consistency, Bijma et al. (2007) suggested that the phenotype of a focal individual $i$ influenced by social interactions (for example, competition) in a group of size $n$ could be modelled as:

$$
\mathrm{y}_{1 \mathrm{i}}=\mathrm{a}_{1 \mathrm{i}}+\sum_{\mathrm{i} \neq \mathrm{j}}^{\mathrm{n}} \mathrm{p}_{1^{\prime} \mathrm{j}}+\varepsilon_{1 \mathrm{i}}=\mathrm{a}_{1 \mathrm{i}}+\sum_{i \neq j}^{n} \mathrm{a}_{1^{\prime} \mathrm{j}}+\sum_{i \neq j}^{n} \varepsilon_{1^{\prime} \mathrm{j}}+\varepsilon_{1 \mathrm{i}}
$$

where $p_{1^{\prime} j}$ is the phenotypic performance of competitor $j$ on the resource-dependent trait $y_{1}$ expressed in focal individual $i$. Though not observed, this performance trait $p_{1^{\prime}}$ in $j$ is assumed to be determined by an additive effect $\left(a_{1^{\prime} j}\right)$ and an environmental deviation from mean performance $\left(\varepsilon_{1^{\prime} j}\right)$. Other terms in Equation 4 are as previously defined. Variance in $\varepsilon_{1^{\prime} j}$ will not be statistically identifiable but under certain assumptions an unbiased estimate of the covariance structure of direct $\left(a_{1}\right)$ and indirect $\left(a_{1^{\prime}}\right)$ genetic effects can nonetheless be estimated (see Bijma et al. (2007) for discussion of group effects) using standard mixed model analyses (for sample code see 'Models for social dominance and competition' at http:// www.wildanimalmodels.org/). An individual's genotype will affect the population mean phenotype through both direct and IGEs. The 'total breeding value' (TBV) of any individual's describes the overall impact of the genotype on the mean of $y_{1}$ (Bijma et al., 2007), and will have a variance of:

$$
\sigma_{\mathrm{TBV} 1}^{2}=\sigma_{\mathrm{A} 1}^{2}+2(\mathrm{n}-1) \cdot \sigma_{\mathrm{A} \cdot 1,1^{\prime}}+(\mathrm{n}-1)^{2} \cdot \sigma_{\mathrm{A} 1^{\prime}}^{2}
$$

where $\sigma_{\mathrm{A} 1}^{2}$ is the direct genetic variance, $\sigma^{2} \mathrm{Al}^{\prime}$ is the indirect genetic variance, $\sigma_{\mathrm{A} .1,1^{\prime}}$ is the direct-indirect genetic covariance and $n$ is group size. The selection response in $y_{1}$ generally depends on this total genetic variance $\sigma_{\mathrm{TBV} 1}^{2}$ as well as on the strength of selection, whether selection is among groups as well as among individuals, and the relatedness structure of the groups (see equation 5 of Bijma et al. (2007) and equation 15 of Bijma and Wade (2008)). However, in the case that competition occurs (to a first approximation) among unrelated individuals and there is no among-group selection, selection response will be determined as the product of $\beta_{1}$ and $\sigma^{2}{ }_{\mathrm{A} 1}+$ $(n-1) \cdot \sigma_{\mathrm{A} \cdot 1,1^{\prime}}$. Competition-based constraint is therefore manifest as a negative value of $\sigma_{\mathrm{A} .1,1^{\prime}}$ (the covariance between direct and IGEs), as this will lead to an expected response $<\beta_{1} \cdot \sigma^{2}{ }_{\mathrm{A} 1}$ (the breeder's equation prediction).

Both trait- and performance-based models allow estimation of IGEs that, coupled with appropriate measures of selection, permit evolutionary environmental deterioration to be incorporated into predictions of phenotypic change (Wolf et al., 1999; Cheverud, 2003). Only the former can be informative for the specific mechanism (that is, trait or traits) of interaction, but the latter may be more widely applicable precisely because there is no need to observe competitive ability. Parameterisation of models that include both trait- and performance-based sources of IGE effects simultaneously should also be both feasible and highly informative. For example, the presence of indirect genetic variance after conditioning focal $y_{1}$ on competitor $y_{2}$ would suggest involvement of further (unknown) traits. Similarly, if $y_{2}$ captures all competitive effects on $y_{1}$, then we expect the genetic correlation between $a_{2}$ and $a_{1^{\prime}}$ to equal 1 .

Typically, it is assumed that interactions occur equally among all individuals within a group (but not between individuals from different groups), and that groups are of equal size (but see Bijma (2010b) and Hadfield and Wilson (2007)). Although design of statistically powerful experiments is challenging (Bijma, 2010a), these conditions can be readily met in experimental studies with groups unambiguously defined as, for example, the set of animals sharing a cage. However, appropriate criteria for defining groups will generally be less clear for field-based studies except where the interaction is dyadic (Brommer and Rattiste, 2008; Teplitsky et al., 2010; discussion 
of social dominance below). In some cases, it may be sensible to define discrete groups based on spatial substructure of a population (for example, if individuals show strong site fidelity). Alternatively, it may be possible to avoid imposing a discrete group structure by adapting methods recently applied to spatially structured forestry trial data, in which the IGE of $j$ on $i$ (that is, $\mathrm{a}_{1^{\prime} j}$ in Equation 4) is weighted by an 'intensity of competition factor' based on the distance separating the trees (Cappa, 2008; Costa e Silva and Kerr, 2013). Use of spatial information in this way also allows explicit modelling of the non-genetic component of indirect competitive effects (that is, $\varepsilon_{1^{\prime} j}$ in Equation 4), which may otherwise bias estimates of indirect genetic variance (Costa e Silva and Kerr, 2013).

For animal studies, analogous intensity factors could be derived from knowledge of the distance between home range centres for territorial species, estimates of resource use overlap (in time and/or space) or pairwise measures of behavioural interaction frequency based on social network analysis (Wey et al., 2008; Sih et al., 2009). Recently, Stopher et al. (2012) investigated the extent to which sharing of fine-scale environmental effects might bias heritability estimates in a wild vertebrate population. To do this they modelled environmental effects on phenotype using two-dimensional autocorrelation structures within an animal model framework, but also defined a matrix of pairwise home range overlap (S) used as an incidence matrix to estimate variance components attributable to a random effect of shared environment. This strategy is appropriate given that positive covariance between neighbours is expected to arise from environmental heterogeneity but would not readily detect the signature of competition (which is expected to induce negative covariance between neighbours). Nonetheless, the individual elements of $\mathbf{S}$ could certainly be used to scale the intensity of competition occurring at both genetic and non-genetic levels within an IGE model.

\section{Conditioning $\mathrm{G}$ on social dominance}

Under the conditions assumed to derive Equation 1, genetic variance in competitive ability $\left(y_{2}\right)$ reduces the evolution of growth rate $\left(y_{1}\right)$. However, the net evolutionary change in $y_{1}$ will still be positive so the constraint on growth rate is not absolute. In contrast, if we assume the population size $(N)$ and total resource $(A)$ are constant then an absolute constraint must exist for the trait of resource acquisition. This is because the mean ration per individual will always be $A / N$. This is a slight over simplification (for example, behavioural or physiological pathways could evolve to permit exploitation of a new resource) but is true to the extent that resource acquisition is determined by the outcome of competition. The nature of this absolute constraint, and its implications, can be most readily understood by considering the case of social dominance as inferred from dyadic contests over a resource.

Dominance is usually defined as the (repeatable) tendency of an individual to win contests, and as it depends causally on resource holding potential (RHP) traits it can be treated as a (potentially) heritable trait (Moore et al., 2002; Wilson et al., 2011) despite arguments to the contrary (Barrette, 1987). As winners gain resource (for example, food, territory, mating opportunities), and ultimately fitness, contest winning is also under positive selection. Although the breeder's equation tells us that a heritable trait under directional selection will evolve, this cannot be true for contest winning as the mean will always be 0.5 (that is, for every winner there is a loser). The constraint arises because the contest outcome for a focal individual $(f)$ depends not only on its own RHP (or dominance) phenotype $(P)$, but also on the effect of its opponent's $(o)$ phenotype $\left(P^{\prime}\right)$. Following the standard quantitative genetic practice of decomposing an individual's phenotypic merit into (additive) genetic breeding values (a) and permanent environment (pe) affects the outcome of some contest for focal individual $F$ could be modelled as:

$$
\text { Outcome }_{\mathrm{fx}}=\mu+\mathrm{P}_{\mathrm{f}}+\mathrm{P}_{\mathrm{o}}^{\prime}+\varepsilon_{\mathrm{X}}=\mu+\mathrm{a}_{\mathrm{Pf}}+\mathrm{pe}_{\mathrm{Pf}}+\mathrm{a}_{\mathrm{P}^{\prime} \mathrm{o}}+\mathrm{pe}_{\mathrm{P}^{\prime} \mathrm{o}}+\varepsilon_{\mathrm{X}}
$$

where $\mu$ is the phenotypic mean (which will equal 0.5 if the outcome is scored as 0/1). In fact, Equation 6 is a special case of more general IGE model of Bijma et al. presented previously (Equation 4), where $n=2$ and additional permanent environment terms are present (which will be identifiable if individuals are observed in multiple contests with different opponents). From Equation 5, it follows that the variance in TBV $\left(\sigma^{2} \mathrm{TBV}\right)$ for dyadic contest outcome is equal to $\sigma_{\text {A.P }}^{2}+2 \sigma_{\text {A.P.P }}+\sigma_{\text {A.P', }}^{2}$ whereas the response to selection among unrelated individuals is determined by $\sigma_{\text {A.P }}^{2}+\sigma_{\text {A.P.P }}$ (where $\sigma_{\text {A.P }}^{2}$ is the additive variance in direct (focal) genetic effects, $\sigma^{2}$ A.P $\mathrm{P}^{\prime}$ is the variance in indirect (opponent) genetic effects and $\sigma_{\mathrm{A} . \mathrm{P}, \mathrm{P}^{\prime}}$ is the covariance between direct and IGEs). However, as any genotype that predisposes to winning when expressed by a focal individual must predispose to losing, and by the same amount, if encountered in an opponent it follows that an individual is direct and indirect genetic merits are of equal magnitude but opposite (that is, $a_{\mathrm{Pi}}=-a_{\mathrm{P}^{\prime} \mathrm{F}}$; Wilson et al., 2011). This implies that $\sigma_{\mathrm{A} . \mathrm{P}}^{2}=\sigma_{\mathrm{A} . \mathrm{P}^{\prime}}=-\sigma_{\mathrm{A} . \mathrm{P}, \mathrm{P}^{\prime}}$ in which case $\sigma^{2} \mathrm{TBV}=0$ (and $\sigma_{\text {A.P }}^{2}+\sigma_{\mathrm{A} . \mathrm{P}, \mathrm{P}^{\prime}}=0$ ). Thus, despite the fact that dominance is heritable, under the IGE modelling framework it becomes apparent that there can be no genetic variance available to facilitate a phenotypic selection response in contest outcome. Although argued logically here, this result has also been obtained empirically from data on dyadic contests between known individuals in a wild population of red deer (Wilson et al., 2011).

Demonstrating that contest outcome cannot evolve is trivial in the sense that we already know this, but does illustrate how ignoring IGEs can lead to nonsensical evolutionary predictions. More practically, if mean contest outcome cannot evolve then genetic variance in life history traits that arises from heritable effects on social dominance will not facilitate selection responses in those traits. Thus, to understand evolutionary potential and constraint in a set of resourcedependent life history traits, we should consider not just the additive genetic covariance matrix among them $(\mathbf{G})$, but also the corresponding matrix conditional on social dominance (subsequently denoted $\mathbf{G}_{\mid \mathrm{D}}$ ).

This can be illustrated with a hypothetical example (Figure 2). Imagine a population in which genetic variance exists for allocation, but not acquisition of some resource that is acquired independently of competition. Different genotypes vary in their relative allocation to survival (S) and fecundity (F) according to a simple trade-off. Now imagine that a secondary resource can also be obtained through contest competition, that there is genetic variance in social dominance (D), and that individuals winning additional resource all allocate it in equal proportions to $\mathrm{S}$ and $\mathrm{F}$ (that is, genetic variance in acquisition but not allocation). The total genetic merits $(a)$ for survival and fecundity, respectively, might then be specified as $a_{\mathrm{F}}=a_{\mathrm{D}}+a_{\mathrm{F} \mid \mathrm{D}}$ and $a_{\mathrm{S}}=a_{\mathrm{D}}+a_{\mathrm{S} \mid \mathrm{D}}$, where $a_{\mathrm{D}}$ is the genetic merit for dominance, and $a_{\mathrm{X} \mid \mathrm{D}}$ is the component of genetic merit for $\mathrm{X}$ that is independent of dominance. Although the covariance between $a_{\mathrm{F} \mid \mathrm{D}}$ and $a_{\mathrm{S} \mid \mathrm{D}}$ will be negative, sufficient variance in $a_{\mathrm{D}}$ will generate a net positive covariance between $a_{\mathrm{F}}$ and $a_{\mathrm{S}}$ (Figure $2 \mathrm{a}$ ).

Eigen decomposition of the full G-matrix among D, S and F does in fact reveal the life-history trade-off in the form of antagonistic loadings on $\mathrm{S}$ and $\mathrm{F}$ associated with vector 2 (Figure $2 \mathrm{~b}$ ). However, the first vector-which explains $>75 \%$ variance in this hypothetical case-has same sign loadings across all three traits. As this vector will 


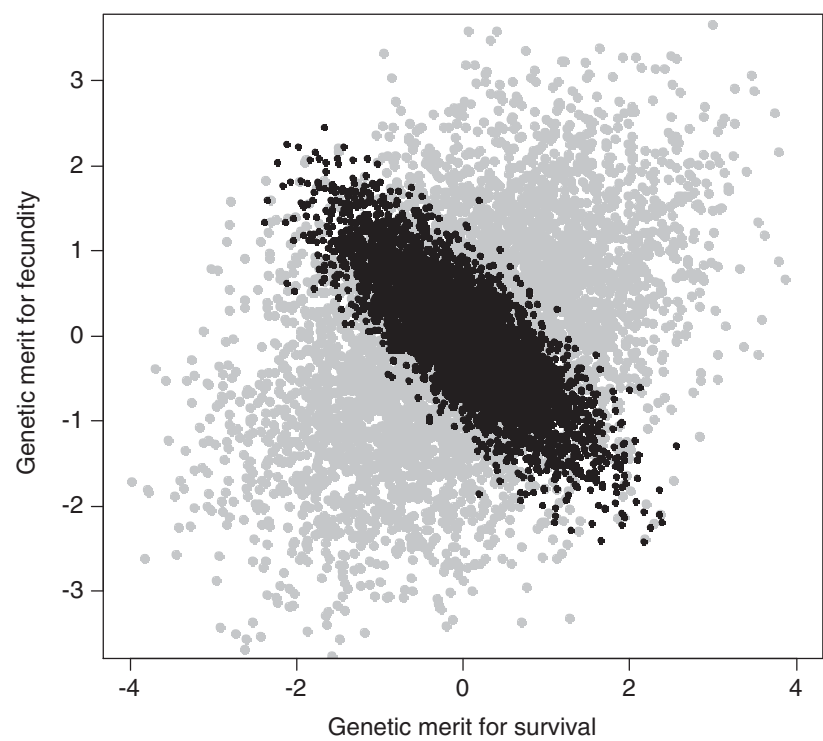

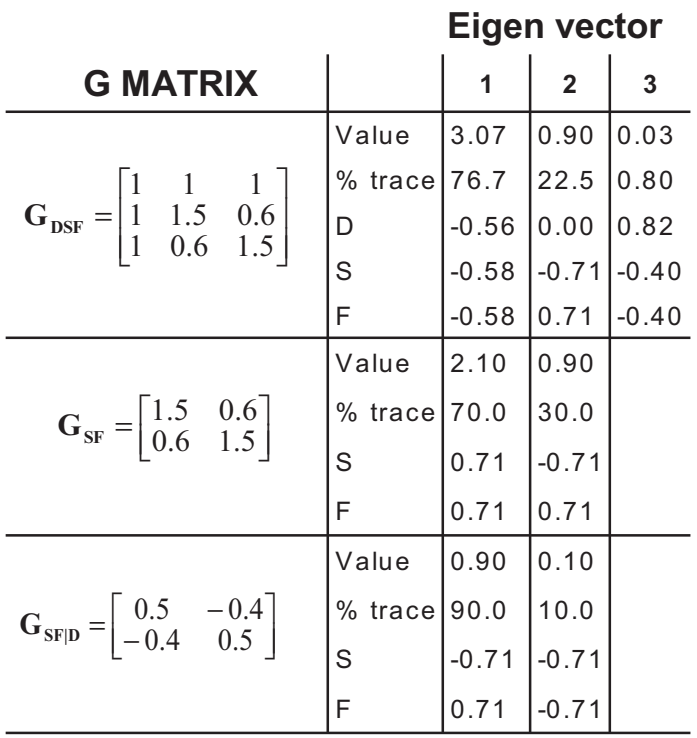

Figure 2 Bivariate distributions of genetic merit for survival (S) and fecundity (F) in a hypothetical population where both traits are resource limited and in a trade-off. Given the genetic variance in resource allocation but not in acquisition, a negative covariance is expected (black dots). However, if resource acquisition (and hence phenotypic expression) is partially dependent on winning contests, sufficient genetic variance for dominance (D) would cause positive covariance between genetic merits for $\mathbf{S}$ and $F$ (grey points). In this case, the structure of the $\mathbf{G}_{\mathrm{DSF}}$ (the $\mathbf{G}-\mathrm{matrix}_{\mathrm{i}}$ defined for $\mathrm{D}$, $\mathbf{S}$ and $\mathrm{F}$ ) or $\mathbf{G}_{\mathrm{SF}}$ (defined for $S$ and $F$ only) would lead a researcher to erroneously expect rapid evolution of increased survival and fecundity. In contrast, the structure of $\mathbf{G}_{\mathrm{SFID}}$ (the variance-covariance matrix for S and F conditional on $\mathrm{D}$ ) indicates most variance accessible to selection is orthogonal to selection, which will be positive on both life history traits.

be (approximately) aligned with selection and could therefore be interpreted as genetic variance in 'quality' (Coltman et al., 2005), one might conclude a rapid selection response is possible. The same conclusion would be reached with knowledge only of the genetic covariance structure between $\mathrm{S}$ and $\mathrm{F}$ (Figure 2b), a situation consistent with most studies of life history traits in which behavioural processes allowing inference of individual dominance status are not observed. If dominance is observed, then recognising that $a_{\mathrm{D}}$ will contribute to phenotypic expression but not a selection response, the covariance structure for $\mathrm{S}$ and $\mathrm{F}$ could be conditioned on $\mathrm{D}$. This conditional matrix (denoted $\mathbf{G}_{\mathrm{SF} \mid \mathrm{D}}$ in Figure $2 \mathrm{~b}$ ) has a first eigen vector that accounts for $90 \%$ of the variance with antagonistic loadings on $\mathrm{S}$ and $\mathrm{F}$. As $\mathrm{S}$ and $\mathrm{F}$ are positively selected, this vector is therefore (approximately) orthogonal to selection and no rapid selection response would be expected.

Here, conditioning on dominance to account for the competitionbased constraint reveals a trade-off between life history components that is manifest as a conditional negative genetic correlation between $\mathrm{S}$ and F. More generally, the importance of competition as constraint could be assessed by substituting conditional genetic parameters into predictive models of selection response. For example, one could compare predictions of the multivariate breeder's equation $\mathbf{R}=\mathbf{G} \boldsymbol{\beta}$ (Lande, 1979) to expectations determined as the product of $\mathbf{G}_{\mid \mathrm{D}}$ and $\boldsymbol{\beta}$. Similarly, if the Robertson-Price identity fails because it does not account for evolutionary environmental deterioration, a better prediction should be obtained by estimating the additive covariance between a trait of interest and relative fitness conditional on dominance. Comparisons of $\mathbf{G}$ and $\mathbf{G}_{\mid \mathrm{D}}$ estimates could usefully allow several further hypotheses to be tested. For instance, if genetic variance in competitive ability prevents detection of trade-offs in $\mathbf{G}$, then application of constraint metrics such as those suggested by Agrawal and Stinchcombe (2009) to $\mathbf{G}_{\mid \mathrm{D}}$ should be revealing. Where these matrices can be estimated under different intensities of competition, one could also test the expectation that $\mathbf{G}$ and $\mathbf{G}_{\mid \mathrm{D}}$ will become more similar with increasing resource and decreasing competition.

Practically, one could estimate G for a set of traits including dominance, and then obtain $\mathrm{G}_{\mid \mathrm{D}}$ following approaches outlined in Hansen et al. (2003). Alternatively, one could estimate $\mathbf{G}_{\mid \mathrm{D}}$ using a multivariate mixed model analysis of phenotypic and pedigree data in which individual dominance status was included as a fixed effect on each trait (Wilson et al., 2009b). Either way obviously requires data informative for (focal) dominance. An important point to stress is that $\mathbf{G}_{\mid \mathrm{D}}$ describes the genetic (co)variance structure among life history traits that is independent of dominance. The argument that it is a better representation of multivariate evolutionary potential than G depends on an assumption of causality-additive covariance between dominance and life history arises because (and only because) expression of life history traits depends on winning contests. In reality, causality could sometimes be reversed (for example, if fastgrowing individuals get big and win contests because size is critical to RHP). Thus, experimental validation of causality is appropriate where it is possible and wider use of path analytic tools (Lynch and Walsh, 1998 ) would be advantageous where it is not.

\section{SUMMARY}

In conclusion, theory tells us that competition could be an important source of evolutionary constraint in natural populations where resources are limiting. The ubiquity of competition, the frequent finding of phenotypic stasis where trait evolution is predicted and the observation that (genetic) covariance among traits in putative tradeoffs is often positive (with respect to effects on fitness) make this hypothesis appealing. However, testing it requires empirical scrutiny of the genetic basis of variation in competitive ability-an aspect of phenotype that has rarely been observed, and for which determining suitable proxies will sometimes be difficult. Here I have outlined several quantitative genetic modelling strategies to address this need. 
However, successful implementation of any strategy will also require an adequate understanding of the behavioural ecology of a system. It seems generally unlikely that one can usefully model the consequences of interacting phenotypes without some biological understanding of those interactions. Thus, although quantifying $\mathbf{G}$ among life history traits is clearly important, our understanding of evolutionary potential and constraint could also benefit greatly by an increased focus on determining the causal pathways by which behavioural processes contribute to this matrix.

\section{CONFLICT OF INTEREST}

The author declares no conflict of interest.

\section{ACKNOWLEDGEMENTS}

This work was supported by a BBSRC David Phillips Fellowship. I am grateful to Craig Walling and Katie Stopher for their comments on an earlier version of this manuscript.

Agrawal AF, Stinchcombe JR (2009). How much do genetic covariances alter the rate of adaptation? Proc Roy Soc B 364: 1593-1605.

Barrette C (1987). Dominance cannot be inherited. Trends Ecol Evol 2: 251.

Bergeron P, Baeta R, Pelletier F, Réale D, Garant D (2010). Individual quality: tautology or biological reality? J Anim Ecol 80: 361-364.

Bergsma R, Kanis E, Knol EF, Bijma P (2008). The contribution of social effects to heritable variation in finishing traits of domestic pigs (Sus scrofa). Genetics 178: 1559-1570.

Bijma P (2010a). Estimating indirect genetic effects: precision of estimates and optimum designs. Genetics 186: 1013-1028.

Bijma P (2010b). Multilevel selection 4: modeling the relationship of indirect genetic effects and group size. Genetics 186: 1029-1031.

Bijma P, Muir WM, Van Arendonk JAM (2007). Multilevel selection 1: quantitative genetics of inheritance and response to selection. Genetics 175: 277-288.

Bijma P, Wade MJ (2008). The joint effects of kin, multilevel selection and indirect genetic effects on response to genetic selection. J Evol Biol 21: 1175-1188.

Biro PA, Stamps J (2008). Are animal personality traits linked to life-history productivity? Trends Ecol Evol 23: 361-368.

Blows MW, Walsh B (2009). Spherical cows grazing in flatland: constraints to selection and adaptation. In: van der Werf J, Graser H-U, Frankham R, Gondro C (eds) Adaptation and Fitness in Animal Populations. Evolutionary and Breeding Perspectives on Genetic Resource Management.

Brommer J, Rattiste K (2008). 'Hidden' reproductive conflict between mates in a wild bird population. Evolution 62: 2326-2333.

Cappa EP (2008). Direct and competition additive effects in tree breeding: Bayesian estimation from an individual tree mixed model. Silvae Genetica 57: 45-56.

Charlesworth B (1990). Optimization models, quantitative genetics, and mutation. Evol Int J Org Evol 44: 520-538.

Charmantier A, Garant D (2005). Environmental quality and evolutionary potential: lessons from wild populations. Proc Roy Soc B 272: 1415-1425.

Cheverud JM (1988). A comparison of genetic and phenotypic correlations. Evolution 42 958-968.

Cheverud JM (2003). Evolution in a genetically heritable social environment. Proc Nat/ Acad Sci USA 100: 4357-4359.

Coltman DW, O'Donoghue P, Hogg JT, Festa-Bianchet M (2005). Selection and genetic (co)variance in bighorn sheep. Evolution 59: 1372-1382.

Cooke F, Taylor PD, Francis CM, Rockwell RF (1990). Directional selection and clutch size in birds. Am Nat 136: 261-267.

Costa e Silva J, Kerr RJ (2013). Accounting for competition in genetic analysis, with particular emphasis on forest genetic trials. Tree Genet Genomes 9: 1-17.

Cresswell W (2001). Relative competitive ability does not change over time in blackbirds. J Anim Ecol 70: 218-227.

de Jong G (1976). A model of competition for food. I. frequency-dependent viabilities. Am Nat 110: 1013-1027.

Dingemanse NJ, Barber I, Wright J, Brommer JE (2012). Quantitative genetics of behavioural reaction norms: genetic correlations between personality and behavioural plasticity vary across stickleback populations. J Evol Biol 25: 485-496.

Dochtermann NA (2011). Testing Cheverud's conjecture: behavioral correlations and behavioral syndromes. Evolution 65: 1814-1820.

Ellegren H, Sheldon BC (2008). Genetic basis of fitness differences in wild populations. Nature 452: 169-175.

Ellen ED, Muir WM, Teuscher F, Bijma P (2007). Genetic improvement of traits affected by interactions among individuals: sib selection schemes. Genetics 176: 489-499.

Fairbairn DJ, Roff DA (2006). The quantitative genetics of sexual dimorphism: assessing the importance of sex-linkage. Heredity 97: 319-328.

Fisher RA (1958). The Genetical Theory of Natural Selection, 2nd edn. Dover Books.
Frank SA, Slatkin M (1992). Fishers fundamental theorem of natural selection. Trends Ecol Evol 7: 92-95.

Griffing B (1967). Selection in reference to biological groups. I. Individual and group selection applied to populations of unordered groups. Aust J Biol Sci 10: 127-139.

Griffing B (1976). Selection in reference to biological groups. VI. Use of extreme forms of nonrandom groups to increase selection efficiency. Genetics 82: 723-731.

Hadfield J (2008). Estimating evolutionary parameters when viability selection is operating. Proc Roy Soc B 275: 723-734.

Hadfield J, Wilson AJ (2007). Multilevel Selection 3: modeling the effects of interacting individuals as a function of group size. Genetics 177: 667-668.

Hadfield JD, Wilson AJ, Kruuk LEB (2011). Cryptic evolution: does environmental deterioration have a genetic basis? Genetics 187: 1099-1113.

Hamel S, Gaillard JM, Festa-Bianchet M, Cote SD (2009). Individual quality, early life conditions, and reproductive success in contrasted populations of large herbivores. Ecology 90: 1981-1995.

Hansen TF, Armbruster WS, Carlson ML, Pelabon C (2003). Evolvability and genetic constraint in Dalechampia blossoms: genetic correlations and conditional evolvability. J Exp Zool 296: 23-39.

House CM, Simmons LW (2012). The genetics of primary and secondary sexual character trade-offs in a horned beetle. J Evol Biol 25: 1711-1717.

Kingsolver JG, Diamond SE (2011). Phenotypic selection in natural populations: what limits directional selection? Am Nat 177: 346-357.

Kingsolver JG, Hoekstra HE, Hoekstra JM, Berrigan D, Vignieri SN, Hill CE et al. (2001). The strength of phenotypic selection in natural populations. Am Nat 157: 245-261.

Kolliker M, Brodie ED, Moore AJ (2005). The coadaptation of parental supply and offspring demand. Am Nat 166: 506-516.

Kruuk LEB, Hadfield J (2007). How to separate genetic and environmental causes of similarity between relatives. J Evol Biol 20: 1890-1903.

Kruuk LEB, Slate J, Pemberton JM, Brotherstone S, Guinness F, Clutton-Brock T (2002). Antler size in red deer: heritability and selection but no evolution. Evolution 56: 1683-1695.

Kruuk LEB, Slate J, Wilson AJ (2008). New answers for old questions: the quantitative genetics of wild animal populations. Annu Rev Ecol Evol Syst 39: 524-548.

Lande R (1979). Quantitative genetic analysis of multivariate evolution, applied to brain: body size allometry. Evolution 33: 402-416.

Lande R, Arnold SJ (1983). The measurement of selection on correlated characters. Evolution 37: 1210-1226

Lush J (1937). Animal breeding plans. lowa State College Press: Ames, lowa.

Lynch M, Walsh B (1998). Genetics and analysis of quantitative traits. Sinauer Associates, Inc.: Sunderland, 980.

McGlothlin JW, Brodie ED III (2009). How to measure indirect genetic effects: the congruence of trait-based and variance-partitioning approaches. Evolution 63: 1785-1795.

McGlothlin JW, Moore AJ, Wolf JB, Brodie ED (2010). Interacting phenotypes and the evolutionary process. III. Social Evolution. Evolution 64: 2558-2574.

Merilä J, Sheldon BC, Kruuk LE (2001). Explaining stasis: microevolutionary studies in natural populations. Genetica 112-113: 199-222.

Moore AJ, Brodie ED, Wolf JB (1997). Interacting phenotypes and the evolutionary process.1. Direct and indirect genetic effects of social interactions. Evolution 51: 1352-1362.

Moore AJ, Haynes KE, Preziosi RF, Moore PJ (2002). The evolution of interacting phenotypes: genetics and evolution of social dominance. Am Nat 160: S186-S197.

Morrissey MB, Kruuk LEB, Wilson AJ (2010). The danger of applying the breeder's equation in observational studies of natural populations. J Evol Biol 23: 2277-2288.

Moyes K, Morgan BJT, Morris A, Morris S, Clutton-Brock TH, Coulson T (2009). Exploring individual quality in a wild population of red deer. J Anim Ecol 78: 406-413.

Muir WM (2005). Incorporation of competitive effects in forest tree or animal breeding programs. Genetics 170: 1247-1259.

Muir WM, Craig JV (1998). Improving animal well-being through genetic selection. Poult Sci 77: 1781-1788.

Nicholson (1954). An outline of the dynamics of animal populations. Aust J Zool 2: 9-65. Parker GA (1974). Assessment strategy and the evolution of fighting behaviour. J Theor Biol 47: 223-243.

Robertson A (1966). A Mathematical Model of Culling Process in Dairy Cattle. Anim Production 8: 95-108.

Roff D (1996). The evolution of genetic correlations: an analysis of patterns. Evolution $\mathbf{5 0}$ $1392-1403$.

Roff DA (2002). Life history evolution. Sinauer Associates, Ltd.: Sunderland.

Rudin FS, Briffa M (2012). Is boldness a Resource Holding Potential trait? Fighting prowess and changes in startle response in the sea anemone Actinia equina. Proc Roy Soc B 279: 1904-1910.

Réale D, Reader SM, Sol D, McDougall PT, Dingemanse NJ (2007). Integrating animal temperament within ecology and evolution. Biol Rev 82: 291-318.

Siepielski AM, DiBattista JD, Carlson SM (2009). It's about time: the temporal dynamics of phenotypic selection in the wild. Ecol Let 12: 1261-1276.

Sih A, Hanser SF, McHugh KA (2009). Social network theory: new insights and issues for behavioral ecologists. Behav Ecol Sociobiol 63: 975-988.

Stearns SC (1989). Trade-offs in life history evolution. Funct Ecol 3: 259-268. 
Stinchcombe JR, Rutter MT, Burdick DS, Tiffin P, Rausher MD, Mauricio R (2002). Testing for environmentally induced bias in phenotypic estimates of natural selection: Theory and practice. Am Nat 160: 511-523.

Stopher KV, Walling CA, Morris A, Guinnesss FE, Clutton-Brock TH, Pemberton JM et al. (2012). Shared spatial effects on quantitative genetic parameters: accounting for spatial autocorrelation and home range overlap reduces estimates of heritability in wild red deer. Evolution 66: 2411-2426.

Teplitsky C, Mills JA, Yarral JW, Merilä J (2010). Indirect genetic effects in a sex-limited trait: the case of breeding time in red-billed gulls. J Evol Biol 23 935-944.

van Noordwijk AJ, de Jong G (1986). Acquisition and allocation of resources: their influence on variation in life history tactics. Am Nat 128: 137-142.

Wey T, Blumstein DT, Shen W, Jordán F (2008). Social network analysis of animal behaviour: a promising tool for the study of sociality. Anim Behav 75: 333-344.
Wilson AJ, Gelin G, Perron M, Réale D (2009a). Indirect genetic effects and the evolution of aggression in a vertebrate system. Proc Roy Soc B 276: 533-541.

Wilson AJ, Morrissey MB, Adams M, Walling CA, Guinness FE, Pemberton JM et al. (2011). Genetics of social dominance in red deer, Cervus elaphus. J Evol Biol 24: 772-783.

Wilson AJ, Nussey DH (2010). What is individual quality? An evolutionary perspective. Trends Ecol Evol 25: 207-214.

Wilson AJ, Pemberton JM, Pilkington JG, Coltman DW, Mifsud DV, Clutton-Brock TH et al. (2006). Environmental coupling of selection and heritability limits evolution. PLOS Bio 4: $1270-1275$.

Wilson AJ, Réale D, Clements MN, Morrissey MB, Postma E, Walling CA et al. (2009b). An ecologist's guide to the animal model. J Anim Ecol 79: 13-26.

Wolf JB (2003). Genetic architecture and evolutionary constraint when the environment contains genes. Proc Natl Acad Sci USA 100: 4655-4660.

Wolf JB, Brodie ED, Moore AJ (1999). Interacting phenotypes and the evolutionary process. II. Selection resulting from social interactions. Am Nat 153: 254-266. 